\title{
Revolutionizing plant biology: multiple ways of genome engineering by CRISPR/Cas
}

\author{
Simon Schiml and Holger Puchta*
}

\begin{abstract}
The precise manipulation of plant genomes relies on the induction of DNA double-strand breaks by site-specific nucleases to initiate DNA repair reactions that are either based on non-homologous end joining (NHEJ) or homologous recombination (HR). Recently, the CRISPR/Cas system emerged as the most important tool for genome engineering due to its simple structure and its applicability to a wide range of organisms. Here, we review the current status of its various applications in plants, where it is used for the successful generation of stable mutations in a steadily growing number of species through NHEJ. Furthermore, tremendous progress in plant genome engineering by HR was obtained by the setup of replicon mediated and in planta gene targeting techniques. Finally, other complex approaches that rely on the induction of more than one DNA lesion at a time such as paired nickases to avoid off-site effects or controlled genomic deletions are beginning to be applied routinely.
\end{abstract}

Keywords: Gene technology, Double-strand break repair, Synthetic nucleases, Targeted mutagenesis, Gene targeting

\section{Background}

Although with the rise of the CRISPR/Cas (clustered regularly interspaced short palindromic repeats and CRISPR-associated) technology double-strand break (DSB)-induced genome engineering moved into the centre of scientific interest in the last 2 years, the basic principles behind this approach were well known for decades. Previous experiments demonstrated that by induction of a unique DSB in plant genomes using a highly specific endonuclease, different types of genome manipulations could be achieved. On one hand, the frequency of gene targeting (GT), the precise integration of a T-DNA via $H R$ with sequences identical to the genomic locus, can be increased by several orders of magnitude [1]. On the other hand, functional open reading frames can be destroyed by imprecise NHEJ [2]. Interestingly, even in the absence of homologies, integration of a T-DNA into a genomic locus by NHEJ can be enhanced by DSB induction [2]. Depending on the location, the induction of two DSBs can result in deletions [3] or reciprocal exchanges between chromosome arms [4].

*Correspondence: holger.puchta@kit.edu

Botanical Institute II, Karlsruhe Institute of Technology, POB 6980, 76049 Karlsruhe, Germany
For many years, these experiments were performed using mega nucleases such as I-SceI $[5,6]$. However, this method required the introduction of the respective target sequence into the plant genome prior to the genome engineering experiment itself. Therefore, targeting endogenous loci is excluded, as engineering the protein for new target sequences is strongly limited [7, 8]. A major improvement was marked by the introduction of zinc finger nucleases (ZFNs) $[9,10]$ : the DNA cleavage domain from the restriction enzyme FokI was fused to the highly variable DNA binding domain (DBD) of a class of zinc finger transcription factors. By combining different zinc fingers in the DBD, different target sites in the DNA are recognized and are cleaved by the nuclease. Unfortunately there are drawbacks to this technique as not all combinations of zinc fingers function well, therefore every new nuclease has to be tested extensively. Furthermore, cloning of a new nuclease is quite time consuming. However, ZFNs are still in use today primarily because there are no open questions regarding their status as intellectual property. The discovery and molecular analysis of transcription activator-like effectors (TALEs) from the plant pathogen species Xanthomonas led to the third important class of engineered nucleases [11]. The TALE DBD consists of numerous repeats, varying only in two amino acid 
residues. In 2009, it was shown that each of the repeating sequences is able to recognize and bind exactly one nucleotide on the DNA $[12,13]$. An engineered TALE nuclease was created by fusing the DBD once again to FokI, in these experiments, cloning of a new nuclease was facilitated by adapting the GoldenGate cloning method [14].

The most recent, yet already the most important type of programmable nucleases utilizes the CRISPR/Cas system. It was originally discovered in the 1980s as a distinctive genomic locus in E. coli $[15,16]$ and was later characterized to serve as an adaptive immune system in many bacteria and archaea [17]. However, the molecular mechanism of the CRISPR/Cas system from Streptococcus pyogenes was not deciphered until 2012 [18]: foreign plasmid or viral DNA entering the bacterial cells are degraded by a single protein, the nuclease Cas9. The target specificity is governed by a short so-called CRISPR-RNA (crRNA), which is encoded in the CRISPR-locus and which is complementary to the invading DNA so that it can bind directly to the foreign DNA using a stretch of 20 nts. An additional short sequence motif next to the target sequence, termed protospacer adjacent motif (PAM), is needed for the correct recognition of the target site. A second short RNA, the trans-activating CRISPR-RNA (tracrRNA), binds to the crRNA, and a stable complex is formed with Cas9. The foreign DNA is then cleaved by two nuclease domains of Cas9. Furthermore, it was shown that the two RNAs can also be fused together to form a so-called single-guide RNA (sgRNA, Fig. 1). At the beginning of 2013, the groups of Feng Zhang at MIT and George Church at Harvard demonstrated that Cas9 can be used for genome engineering in human cell cultures, proving that it also works in eukaryotic systems $[19,20]$. Since then, the CRISPR/ Cas system has made unprecedented success as a tool for genome engineering due to its ease in cloning new sequence-specific nucleases and the fact that it works in almost any organism. Here, we review the major technical advances that have been made with Cas9 in plants.

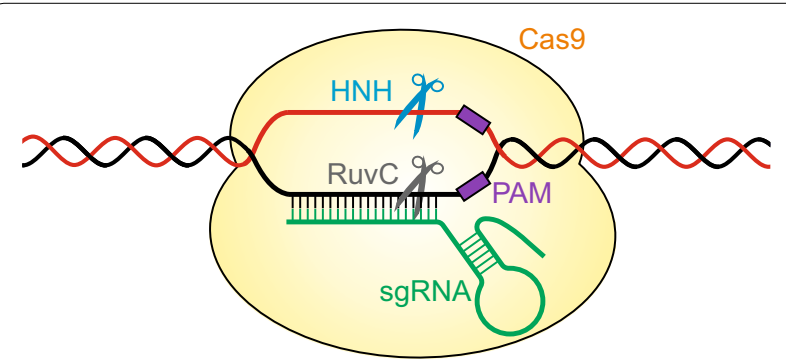

Fig. 1 Schematic representation of the Cas9 cleavage mechanism. The Cas9/sgRNA complex recognizes and binds the complementary sequence next to the PAM, which is highly specific for each Cas9 from different bacterial species. The RuvC domain and HNH motif of Cas9 cleave the two DNA strands 3 bp upstream of the PAM

\section{Creating heritable mutations with RNA-guided Cas9 in plants}

In August 2013, three studies were published in the same issue of nature biotechnology showing that Cas9 also works in plant cells. Scientists from the Chinese Academy of Sciences in Beijing demonstrated the disruption of endogenous genes in rice and wheat protoplasts as well as in rice calli [21] while researchers from Harvard reported cleavage activity of Cas9 in protoplasts of Arabidopsis thaliana and Nicotiana benthamiana [22]. Finally, the Sainsbury Lab in the UK used $N$. benthamiana leaf tissue for agroinfiltration, demonstrating Cas9-mediated disruption of the PDS gene (phytoene desaturase) [23].

However, stable inheritance of Cas9-mediated mutations in natural genes was not reported until 2014, when Feng et al. used the human codon-optimized Cas9 (hCas9) from F. Zhang [19] under the control of two CaMV 35S promoters and 12 different sgRNAs driven by the AtU6-26 promoter to target endogenous sites in A. thaliana [24]. U6 promoters control transcription by RNA polymerase III, which is specific for the production of short non-coding RNAs. The Cas9-harbouring constructs were stably integrated through Agrobacterium tumefaciens. T1 analyses revealed high mutation frequencies ranging from $30 \%$ up to $92 \%$ with 1-bp insertions being the dominant type of mutation. Investigation of T2 and T3 generations revealed Mendelian segregation and stable inheritance of mutations together with the rise of new somatic mutations, which are a result of ongoing DSB induction by the presence of the nuclease transgene. By using an interrupted GUS reporter construct [25], the ability of Cas9 to induce HR was also shown. By whole genome sequencing and Sanger sequencing of potential off-target sites, it was also demonstrated that the system is highly specific in plants as no significant off-target activity was detected. The same group also reported the generation of mutants for a set of genes in rice [26]. By transforming calli with subsequent regeneration of transgenic T0 plants, Cas 9 activity could be observed early in plant development, leading to a high number of mutated plants. Additionally, heredity of some mutations to the T1 generation was demonstrated but not analysed in detail due to a high number of secondary mutations that created chimeric plants.

Soon after the reports mentioned above, the use of a distinct construct in A. thaliana was reported [27]. A codon optimized version of Cas9 is controlled by a plant Ubiquitin promoter, the sgRNA is expressed by the AtU6-26 promoter and both elements are located on the same T-DNA. Following stable transformation, homozygous mutations were induced at a frequency of up to $70 \%$ in two endogenous marker genes of $A$. thaliana and in addition, inheritance of mutations into T2 and 
T3 generations as well as their segregation in a Mendelian fashion was demonstrated. Mutation patterns were analysed by next generation sequencing, which again exhibited small insertions of 1-bp to be the most dominant form of alteration. Furthermore, the first application of the Cas9-D10A nickase variant to plants was reported here. By inducing a point mutation to Cas9 that inactivates one of the nuclease domains, the nuclease is converted to a single-strand break (SSB) inducing nickase. NGS did not reveal a mutagenic potential of the nickase. However, when the constructs were applied to the HR reporter plant lines DGU.US and IU.GUS [28], the nickase was able to induce HR at least as efficiently as the Cas9 nuclease, rendering it a promising tool for HRbased genome engineering approaches.

Zhou et al. presented a study on the application of RNA-guided Cas9 in rice [29]. Here, a rice codon-optimized Cas9 was put under control of a maize ubiquitin promoter. Notably, two different sgRNAs, that have successfully been used in the mammalian system before (one with $48 \mathrm{nt}$ tracr-tail [19] and one with $85 \mathrm{nt}$ tracr tail [20]), as well as a dual guide system (dgRNA) with separate crRNA and tracrRNA, were used all under the control of a rice U6 promoter. When stably transformed to rice plants, the $48 \mathrm{nt}$ sgRNA did not induce any detectable mutations, while the induction by the dgRNA was very low in the T0 generation ( 2 out of 16 transformants with a mono-allelic mutation). One might speculate, that these RNA constructs are not able to form a stable RNACas9 complex under plant growth conditions. However, the $85 \mathrm{nt}$ sgRNA did induce NHEJ events in the transformed generation with efficiencies ranging from $20 \%$ up to $100 \%$ of a small number of transformants. All of the mutations observed were already bi-allelic and the two mutated alleles showed Mendelian segregation in T1 and in transgene-free T2 plants. Furthermore, the induction of large deletions of up to $245 \mathrm{~kb}$ was observed in transgenic plants when two sgRNAs were applied, although inheritance was not investigated in this approach.

The van Eck group at the Cold Spring Harbor Laboratory was the first to demonstrate Cas9-mediated heritable mutations in tomato [30]. The hCas9 driven by the $35 \mathrm{~S}$ promoter was combined with two sgRNAs under AtU6 control, intending to create a specific deletion in the SlAGO7 gene for easy detection of mutations. Almost half of all transformants exhibited the recessive wiry2-1 growth phenotype [31], indicating homozygous mutations early in development. Sanger sequencing confirmed the presence of respective mutations. However, in addition to the expected deletion, larger deletions that included the target size were observed, as well as small mutations affecting one or both of the target sequences. To show inheritance of mutations, wild-type flowers were pollinated by the mutant plants and the offspring were analysed. Heterozygous plants without the Cas9 transgene but with one wild-type and one mutant allele could be identified, thus confirming heredity of the mutations.

The group of Qi-Jun Chen from Beijing applied their RNA-guided Cas9 to A. thaliana and maize [32]. Using a set of different constructs (maize codon-optimised zCas9 or hCas9, Ubiquitin or $2 \times 35 \mathrm{~S}$ promoter, AtU6-26, OsU3 or TaU3 promoter for the sgRNA), targeted mutagenesis was demonstrated in both maize protoplasts and transgenic plants, with the combination of zCas9 and TaU3p exhibiting the highest efficiency. By using different Pol III promoters, it was also possible to assemble up to four different sgRNA expression cassettes on one vector for multiplex genome engineering. Heritability of mutations was confirmed in A. thaliana by checking for transgenefree T2 plants via PCR and subsequent sequencing of the Cas9 target sites. At the moment, the number of reports of new stably mutated plant species using CRISPR/Cas is rapidly growing $[33,34]$.

\section{Harnessing different Cas9 orthologues}

A major step towards more complex applications of the CRISPR/Cas system was made by the adaption of additional Cas9 nucleases to genome engineering. The most widely used Cas9 nuclease originates from Streptococcus pyogenes. However, it was shown in mammalian cell culture that Cas9 orthologues from other species are also applicable for targeting unique genomic sites [35, 36]. Recently, it was shown that nucleases from Streptococcus thermophilus and Staphylococcus aureus also work efficiently in A. thaliana [37]. Stably transformed constructs contained a Cas9 expression system together with the species-specific sgRNA with distinct PAM-specificities. It was demonstrated that both nucleases led to highly efficient mutagenesis. For S. aureus, Cas9 targeting a specific PAM ('NNGGGT') increased the mutation frequency to almost $90 \%$, with the most dominant form of mutations being deletions. Mutations in the $A D H 1$ locus were shown to be heritable for both nucleases. Furthermore, it was demonstrated that cross interferences between Cas9 and sgRNA from different species do not occur. These findings provide the basis for more complex approaches, enabling the simultaneous control of different enzymatic activities in a single plant cell [38].

\section{Inducing genomic change during plant and organ development}

Although the use of constitutive promoters exhibits high frequency mutagenesis for Cas9, other promoters are also desirable. This offers the possibility to achieve stable mutations more quickly, especially for plant species with long generation times. Additionally, conditional knockout 
or the mutagenesis of specific genes in unique organs is rendered possible by the use of respective promoters.

Together with scientists from Korea, George Coupland showed high-frequency mutagenesis in A. thaliana [39]. In their construct, instead of a constitutive promoter, the hCas 9 was controlled by the INCURVATA 2 promoter, which is highly active in meristematic tissue. For the sgRNA, the AtU6-26 promoter was used. Mutagenesis frequencies for three endogenous targets ranged from $10 \%$ to $\sim 85 \%$, and T2 and T3 analysis revealed segregation of certain mutations as expected along with the induction of new mutations in the respective generation.

Qi-Jun Chen's group from Beijing reported a similar experiment [40]: the zCas9 was put under control of the promoter from the egg cell-specific EC1.2 of A. thaliana to increase heritability by inducing mutations in egg cells. Together with respective sgRNAs, T1 double and triple mutant Arabidopsis plants could be obtained that also segregated in the T2 generation. Furthermore, different combinations out of eight promoters and two terminators were analysed for their mutagenesis efficiency. The highest number of $\mathrm{T} 1$ triple mutants $(17 \%)$ was observed using a combination of the EC1.2 enhancer and EC1.1 promoter together with the $r b c S E 9$ terminator. Although this approach does not seem to significantly increase heritability of mutations compared to using a somatic expression system, having the ability to control mutagenesis through different developmental stages can be of great importance for studying the function of certain genes.

\section{Off-site effects and how to avoid them}

A major concern when using an RNA-guided Cas9 is off-site activity. Although extensive studies that address this issue have been performed in the bacterial and mammalian system, the exact extent of off-site activity is still not completely clear. For plants, there is little data currently available that addresses off-site activity. Sequencing of bioinformatically identified putative off-target sites showed no detectable events in A. thaliana, $N$. benthamiana, wheat, rice and sweet orange [21-24, 29, 41, 42]. Whole-genome sequencing of mutated $A$. thaliana plants also resulted in no off-target events [24]. In contrast to these results, a study using Cas 9 in rice found a putative off-target site to be mutated in $1.6 \%$ of the investigated plants, although this was still five times less frequent than the on-target site [43]. A study covering the application of Cas9 to soybean reported an off-target frequency of $13 \%$ [44]. It has to be noted, however, that in this study, two paralogues of DDM1 were targeted and therefore the high sequence similarity is likely to cause the high amount of off-target activity.

A solution to off-target activity proposed by experiments in human cell culture is the use of two Cas 9 nickases [45]. By introducing a point mutation (D10A) into one of the nuclease domains of Cas9, the enzyme is converted into a SSB inducing nickase. The nickase can then be guided to two adjacent positions in the genome by two distinct sgRNAs resulting in SSBs on each of the two DNA strands (Fig. 2). The result is a DSB that can also lead to NHEJ-mediated mutagenesis. Hence, the
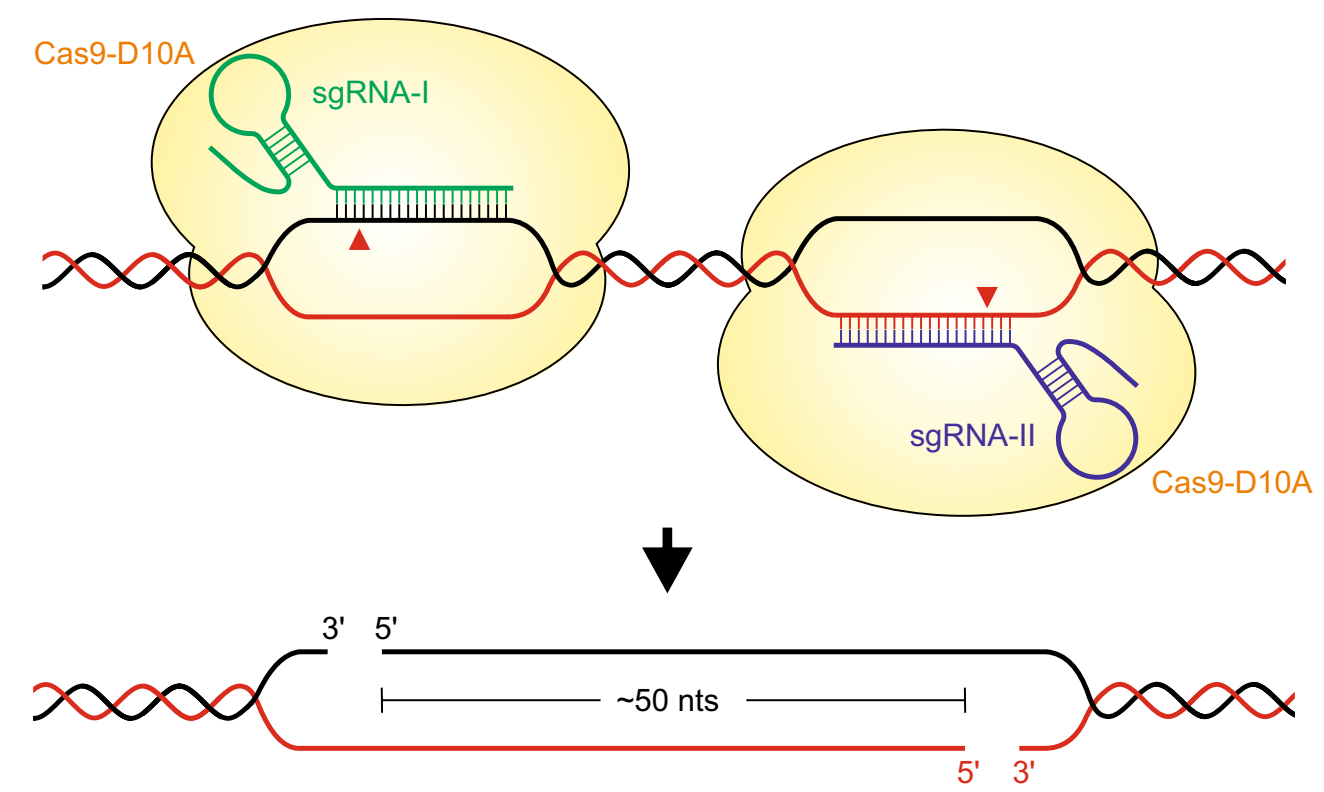

Fig. 2 Cas9 paired nickases approach. By using 2 sgRNAs, the D10A nickase variant can be guided to the two opposite DNA strands at adjacent positions. The resulting BSD exhibits long single-stranded 5'-overhangs 
specificity is increased, as independent off-site binding of the sgRNAs does not lead to mutations. It has already been demonstrated that this approach is also applicable to plants. By adding a second sgRNA to a Cas9 construct and subsequent stable transformation into A. thaliana, a mutagenesis rate comparable to that of the Cas9 single nuclease was achieved [46]. Notably, the mutation pattern shifted from small insertions for the nuclease to larger deletions being the most dominant mutagenesis outcome. Again, mutations were proven to be heritable by demonstrating the presence of mutations in transgene-free T2 plants.

\section{Utilizing homologous recombination}

Site-specific integration of transgenes or precise genome alterations (referred to hereafter as gene targeting, GT) have always been major challenges in plant genome engineering. This is because NHEJ is by far the preferred mechanism to repair DNA breaks in somatic plant tissue. However, using the different classes of engineered nucleases, a wide variety of successful GT experiments have been performed [47]. A major step was taken with the development of the I-SceI-based in planta GT system
[48], which allowed for GT rates of more than $1 \%$ in $A$. thaliana without having to rely on high transformation rates. Using Cas9, it was possible to improve this technique even further [46]: the number of T-DNAs needed for the system to work was reduced from three (donor sequence, artificial I-SceI target sequence and I-SceI expression system) to only one, harbouring both the donor sequence and the Cas9/sgRNA expression cassettes. Furthermore, the system's target flexibility allowed for the targeting of an endogenous locus in the Arabidopsis genome. The precise site-specific integration of the donor sequence into this locus and the possible inheritance of this manipulation into the next generation were demonstrated by using the experimental setup shown in Fig. 3.

In an innovative approach, the group of Dan Voytas combined Cas9-mediated mutagenesis and GT with geminivirus-based replicons [49]. Arabidopsis plants were stably transformed with a specific T-DNA flanked by the viral large intergenic regions (LIRs). Upon cotransformation of the viral replication-initiation protein, replicational release, circularisation and rolling-circle replication of the replicon is initiated at the LIRs. The

endogenous target locus
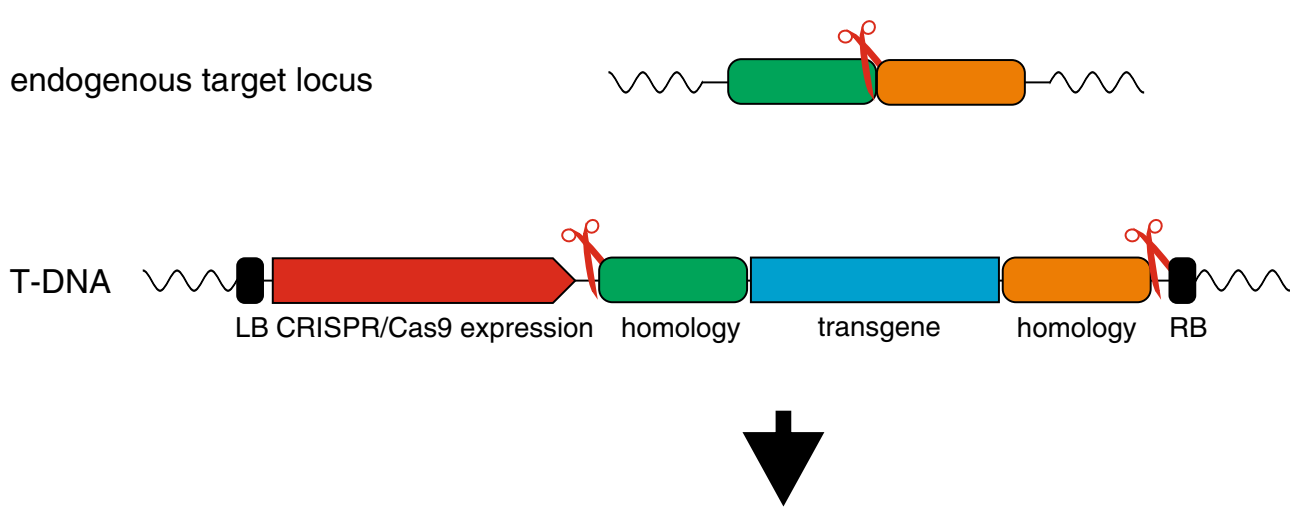

released GT vector

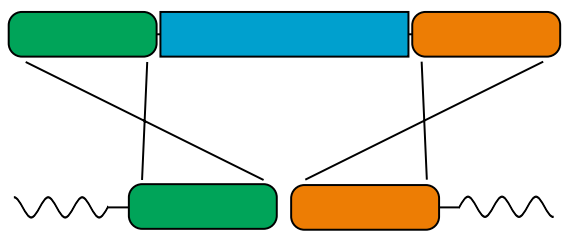

$\downarrow$

heritable GT event

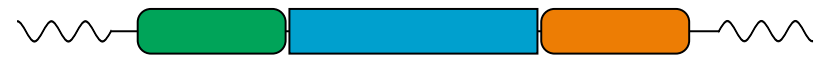

Fig. 3 Overview of the Cas9-mediated in planta GT system. The nuclease and the DNA donor sequence are located on one T-DNA that is stably transformed into the plant. The nuclease induces two DSBs that release the donor intermediate and a third DSB that activates the target locus for $\mathrm{HR}$. The donor sequence integrates into the target locus by using the flanking homologous regions 
circularisation leads to two $35 \mathrm{~S}$ promoters becoming correctly oriented in front of the desired gene, i.e., the nuclease ORF. With this strategy, NHEJ-mediated mutagenesis was demonstrated with ZFNs, TALENs and Cas9. Furthermore, it was shown that when a GT donor sequence was added to the replicon, ZFN-mediated GT could be achieved (Fig. 4). This strategy was also shown to be applicable using Cas9 in tomato [50].

Recently, scientists from DuPont Pioneer reported CRISPR/Cas-mediated GT in soybean [34]. Cas9-sgRNA constructs and a donor template were co-transformed into embryonic callus by particle bombardment. For two target sites on chromosome 4 , the correct integration of the hygromycin resistance gene $H P T$ was identified by PCR and confirmed by Southern blot in T0, and respective plants were regenerated. For one target in T1, three plants with the correct GT event and without any additional transgenes could be identified, confirming inheritance of the new allele. For the second target, only events with additional integration of either Cas9 at the target site or the donor template at random genomic sites were isolated. Notably, the amount of donor- and sgRNACas9-DNA was not optimised to obtain true GT events. Furthermore, as this was a proof-of-principle study, only a small number of events was regenerated, and a true GT event for the second target is likely to be found when analysing a higher number of events.

\section{Multiplex genome engineering}

The architecture of the CRISPR/Cas system with the constant Cas9 protein and the sgRNA-derived target specificity provides the opportunity to target multiple sites at once, as is the case in the natural bacterial system. However, we are only beginning to exploit this possibility, and successful imitation of the bacterial system with polycistronic crRNAs and tracrRNAs has not yet been reported for plants. Therefore, the most common approach by some groups to achieve multiplex sgRNA expression is

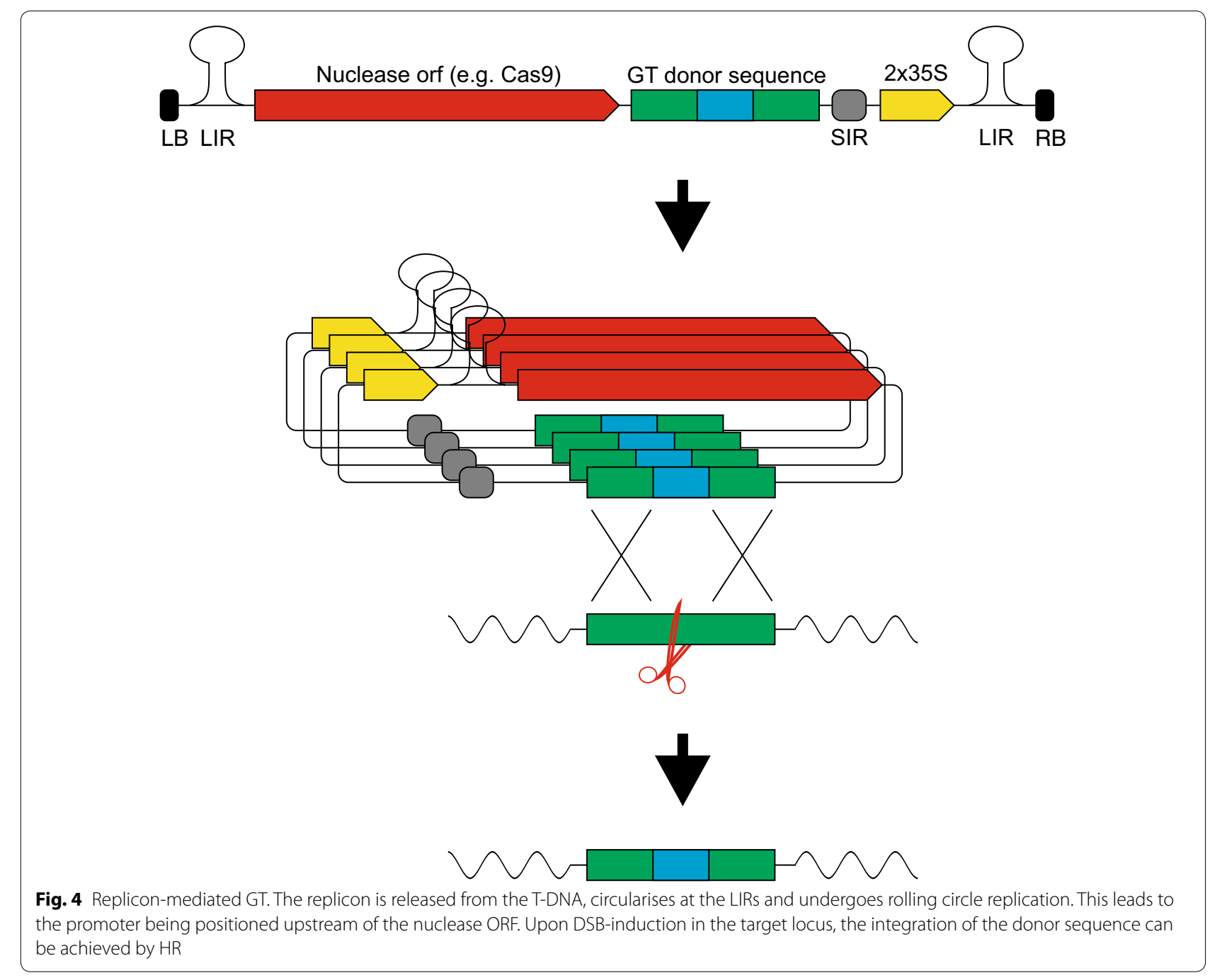


to simply assemble numerous sgRNA expression systems, each with its own promoter [32, 46, 51]. However, this method is limited as constructs become very large with a Pol III promoter for every sgRNA. Xie et al. developed a clever solution to overcome this problem [52]: tRNA sequences were put in between the sgRNA sequences on the construct to create a single polycistronic gene. Two host-endogenous nucleases cleave the expressed RNA at the borders between tRNA and sgRNA creating individual sgRNAs. Simultaneous multiplex mutagenesis was demonstrated in rice protoplasts and transgenic plants for different numbers of sgRNAs (up to eight) in a tRNAsgRNA array.

Scientists from KAUST in Saudi Arabia demonstrated the potential of plant viruses for multiplex genome engineering [53]. In their study, the Tobacco rattle virus (TRV) was used to deliver sgRNAs to transgenic $N$. benthamiana that stably overexpressed Cas9. The two TRV RNAs were introduced into the plants by leaf agroinfiltration with a mixture of two bacterium cultures, one with a plasmid for RNA1, one for RNA2. The latter contained the sgRNA expression system. After reconstitution of the TRV in the infiltrated tissue, a systemic infection throughout the plant leads to sgRNA expression in all tissues and therefore efficient mutagenesis. By mixing bacterial cultures with different RNA2 vectors, simultaneous mutagenesis of two loci was achieved, indicating the potential for multiplex genome engineering by TRV-mediated sgRNA delivery.

\section{Controlling transcription with Cas9}

Targeted genome manipulations with RNA-guided Cas9 have not only been achieved by altering the genome itself but also by influencing transcription. By guiding a catalytically inactive dead (d) Cas9 to a promoter or coding region, transcription can be efficiently blocked [54]. This effect can be enhanced by fusing a repressor protein such as the KRAB domain to dCas9. Likewise, an activator such as VP64 can also be fused to targeted dCas9 to activate transcription of a specific gene [55].

Magdy Mahfouz's group from KAUST was able to transfer this system to plants [56]. The C-terminus of the human codon-optimized dCas9 was fused to the EDLL domain [57] or to the TAL activation domain [58] to create artificial activators. A repressor was created by fusing the SRDX EAR motif [59]. Successful transcriptional activation or repression could be observed in infiltrated $N$. benthamiana leaves by measuring expression levels of a transient GUS gene or the endogenous PDS. Activation was highest when the activator was guided to the sense strand of the promoter near the transcriptional start site and both activator constructs performed at a comparable level. Repression of PDS was demonstrated for both
dCas9 alone and for the dCas9: SRDX fusion construct and could be increased by guiding the complex to several target sites within the promoter and the first exon of the gene simultaneously.

\section{Conclusions and outlook}

In the last 2 years, CRISPR/Cas has emerged as the most important tool for molecular biology due to its simplicity, versatility and efficiency. The immediate benefit for plant scientists is the possibility to rapidly create mutations in genes where no known T-DNA insertion or EMS mutant is available. Use of this method will therefore lead to a more complete understanding of gene function in plants. This approach can not only be applied to genes with unknown functions but also to genes for which we must revise our current knowledge due to the option to produce true knock-out mutants. For instance, scientists from UCSD recently demonstrated that a putative key player of auxin signalling in Arabidopsis $(A B P 1)$ does not possess the important function that was inferred from the analysis of plants obtained by more classical mutagenesis techniques [60]. However, NHEJ-mediated mutation is only a first, yet important step. Specific changes of single amino acids or integration of a larger piece of DNA in the plant genome can be achieved by using Cas9-based GT systems, while efficient multiplex systems will allow the complex rearrangement of chromosomes. Thus, as well as coming closer to developing synthetic plant genomes, we will be also able to obtain plants with a single engineered point mutation that cannot be discriminated from natural varieties. Such plants can even be obtained without the use of transgenic DNA [61], which will also help accelerate the acceptance of Cas9 mutagenized crop plants for agricultural use in the general public.

\section{Authors' contributions}

SS and HP wrote the manuscript. Both the authors read and approved the final manuscript.

\section{Acknowledgements}

We apologize to all colleagues in the field for not being able to cite all of the rapidly growing number of reports published on the use of the RNA-guided Cas9 technology in plants due to space limitations. Furthermore, we want to thank our colleagues Friedrich Fauser and Jeannette Steinert for sharing projects and enthusiasm for Cas9-mediated genome engineering in our group.

\section{Competing interests}

The authors declares that they have no competing interests.

Received: 5 November 2015 Accepted: 5 January 2016

Published online: 28 January 2016

\section{References}

1. Puchta H, Dujon B, Hohn B. Two different but related mechanisms are used in plants for the repair of genomic double-strand breaks by homologous recombination. Proc Natl Acad Sci USA. 1996;93:5055-60. 
2. Salomon S, Puchta H. Capture of genomic and T-DNA sequences during double-strand break repair in somatic plant cells. EMBO J. 1998;17:608695. doi:10.1093/emboj/17.20.6086.

3. Siebert R, Puchta H. Efficient repair of genomic double-strand breaks by homologous recombination between directly repeated sequences in the plant genome. Plant Cell. 2002;14:1121-31.

4. Pacher M, Schmidt-Puchta W, Puchta H. Two unlinked doublestrand breaks can induce reciprocal exchanges in plant genomes via homologous recombination and nonhomologous end joining. Genetics. 2007;175:21-9. doi:10.1534/genetics.106.065185.

5. Puchta H. The repair of double-strand breaks in plants: mechanisms and consequences for genome evolution. J Exp Bot. 2005;56:1-14. doi:10.1093/jxb/eri025.

6. Jacquier A, Dujon B. An intron-encoded protein is active in a gene conversion process that spreads an intron into a mitochondrial gene. Cell. 1985;41:383-94.

7. Steuer S, Pingoud V, Pingoud A, Wende W. Chimeras of the homing endonuclease PI-Scel and the homologous Candida tropicalis intein: a study to explore the possibility of exchanging DNA-binding modules to obtain highly specific endonucleases with altered specificity. Chem Biochem. 2004;5:206-13. doi:10.1002/cbic.200300718.

8. Gimble FS, Moure CM, Posey KL. Assessing the plasticity of DNA target site recognition of the PI-Scel homing endonuclease using a bacterial two-hybrid selection system. J Mol Biol. 2003;334:993-1008.

9. Kim YG, Cha J, Chandrasegaran S. Hybrid restriction enzymes: zinc finger fusions to Fok I cleavage domain. Proc Natl Acad Sci USA. 1996;93:1156-60.

10. Smith J, Bibikova M, Whitby FG, Reddy AR, Chandrasegaran S, Carroll $D$. Requirements for double-strand cleavage by chimeric restriction enzymes with zinc finger DNA-recognition domains. Nucleic Acids Res. 2000;28:3361-9.

11. Bonas U, Stall RE, Staskawicz B. Genetic and structural characterization of the avirulence gene avrBs3 from Xanthomonas campestris pv. vesicatoria. Mol Gen Genet. 1989;218:127-36.

12. Boch J, Scholze H, Schornack S, Landgraf A, Hahn S, Kay S, et al. Breaking the code of DNA binding specificity of TAL-type III effectors. Science. 2009:326:1509-12. doi:10.1126/science.1178811.

13. Moscou MJ, Bogdanove AJ. A simple cipher governs DNA recognition by TAL effectors. Science. 2009;326:1501. doi:10.1126/science.1178817.

14. Cermak T, Doyle EL, Christian M, Wang L, Zhang Y, Schmidt C, et al. Efficient design and assembly of custom TALEN and other TAL effectorbased constructs for DNA targeting. Nucleic Acids Res. 2011;39:e82. doi:10.1093/nar/gkr218.

15. Stern MJ, Ames GF, Smith NH, Robinson EC, Higgins CF. Repetitive extragenic palindromic sequences: a major component of the bacterial genome. Cell. 1984;37:1015-26.

16. Ishino Y, Shinagawa H, Makino K, Amemura M, Nakata A. Nucleotide sequence of the iap gene, responsible for alkaline phosphatase isozyme conversion in Escherichia coli, and identification of the gene product. J Bacteriol. 1987;169:5429-33.

17. Wiedenheft B, Sternberg SH, Doudna JA. RNA-guided genetic silencing systems in bacteria and archaea. Nature. 2012;482:331-8. doi:10.1038/ nature10886.

18. Jinek M, Chylinski K, Fonfara I, Hauer M, Doudna JA, Charpentier E. A programmable dual-RNA-guided DNA endonuclease in adaptive bacterial immunity. Science. 2012;337:816-21. doi:10.1126/science.1225829.

19. Cong L, Ran FA, Cox D, Lin S, Barretto R, Habib N, et al. Multiplex genome engineering using CRISPR/Cas systems. Science. 2013;339:819-23. doi:10.1126/science.1231143.

20. Mali P, Yang L, Esvelt KM, Aach J, Guell M, DiCarlo JE, et al. RNA-guided human genome engineering via Cas9. Science. 2013;339:823-6. doi:10.1126/science.1232033.

21. Shan Q, Wang Y, Li J, Zhang Y, Chen K, Liang Z, et al. Targeted genome modification of crop plants using a CRISPR-Cas system. Nat Biotechnol. 2013;31:686-8. doi:10.1038/nbt.2650

22. Li J, Norville JE, Aach J, McCormack M, Zhang D, Bush J, et al. Multiplex and homologous recombination-mediated genome editing in Arabidopsis and Nicotiana benthamiana using guide RNA and Cas9. Nat Biotechnol. 2013;31:688-91. doi:10.1038/nbt.2654.

23. Nekrasov V, Staskawicz B, Weigel D. Jones, Jonathan D G, Kamoun S. Targeted mutagenesis in the model plant Nicotiana benthamiana using Cas9 RNA-guided endonuclease. Nat Biotechnol. 2013;31:691-3. doi:10.1038/nbt.2655.

24. Feng Z, Mao Y, Xu N, Zhang B, Wei P, Yang D, et al. Multigeneration analysis reveals the inheritance, specificity, and patterns of CRISPR/ Cas-induced gene modifications in Arabidopsis. Proc Natl Acad Sci USA. 2014;111:4632-7. doi:10.1073/pnas.1400822111.

25. Mao Y, Zhang H, Xu N, Zhang B, Gou F, Zhu J. Application of the CRISPRCas system for efficient genome engineering in plants. Mol Plant. 2013;6:2008-11. doi:10.1093/mp/sst121.

26. Zhang H, Zhang J, Wei P, Zhang B, Gou F, Feng Z, et al. The CRISPR/Cas9 system produces specific and homozygous targeted gene editing in rice in one generation. Plant Biotechnol J. 2014;12:797-807. doi:10.1111/ pbi. 12200.

27. Fauser F, Schiml S, Puchta H. Both CRISPR/Cas-based nucleases and nickases can be used efficiently for genome engineering in Arabidopsis thaliana. Plant J. 2014;79:348-59. doi:10.1111/tpj.12554.

28. Orel N, Kyryk A, Puchta H. Different pathways of homologous recombination are used for the repair of double-strand breaks within tandemly arranged sequences in the plant genome. Plant J. 2003;35:604-12.

29. Zhou H, Liu B, Weeks DP, Spalding MH, Yang B. Large chromosomal deletions and heritable small genetic changes induced by CRISPR/Cas9 in rice. Nucleic Acids Res. 2014;42:10903-14. doi:10.1093/nar/gku806.

30. Brooks C, Nekrasov V, Lippman ZB, van Eck J. Efficient gene editing in tomato in the first generation using the clustered regularly interspaced short palindromic repeats/CRISPR-associated9 system. Plant Physiol. 2014;166:1292-7. doi:10.1104/pp.114.247577.

31. Yifhar T, Pekker I, Peled D, Friedlander G, Pistunov A, Sabban M, et al. Failure of the tomato trans-acting short interfering RNA program to regulate AUXIN RESPONSE FACTOR3 and ARF4 underlies the wiry leaf syndrome. Plant Cell. 2012;24:3575-89. doi:10.1105/tpc.112.100222.

32. Xing H, Dong L, Wang Z, Zhang H, Han C, Liu B, et al. A CRISPR/ Cas9 toolkit for multiplex genome editing in plants. BMC Plant Biol. 2014;14:327. doi:10.1186/s12870-014-0327-y.

33. Schaeffer SM, Nakata PA. CRISPR/Cas9-mediated genome editing and gene replacement in plants: transitioning from lab to field. Plant Sci. 2015;240:130-42. doi:10.1016/j.plantsci.2015.09.011.

34. Li Z, Liu Z, Xing A, Moon BP, Koellhoffer JP, Huang L, et al. Cas9-guide RNA directed genome editing in soybean. Plant Physiol. 2015. doi:10.1104/ pp.15.00783.

35. Ran FA, Cong L, Yan WX, Scott DA, Gootenberg JS, Kriz AJ, et al. In vivo genome editing using Staphylococcus aureus Cas9. Nature. 2015;520:18691. doi:10.1038/nature14299.

36. Esvelt KM, Mali P, Braff JL, Moosburner M, Yaung SJ, Church GM. Orthogonal Cas9 proteins for RNA-guided gene regulation and editing. Nat Methods. 2013;10:1116-21. doi:10.1038/nmeth.2681.

37. Steinert J, Schiml S, Fauser F, Puchta H. Highly efficient heritable plant genome engineering using Cas9 orthologues from Streptococcus thermophilus and Staphylococcus aureus. Plant J. 2015.

38. Puchta H. Using CRISPR/Cas in three dimensions. Towards synthetic plant genomes, transcriptomes and epigenomes. Plant J. 2015.

39. Hyun Y, Kim J, Cho SW, Choi Y, Kim J, Coupland G. Site-directed mutagenesis in Arabidopsis thaliana using dividing tissue-targeted RGEN of the CRISPR/Cas system to generate heritable null alleles. Planta. 2015;241:271-84. doi:1 0.1007/s00425-014-2180-5.

40. Wang Z, Xing H, Dong L, Zhang H, Han C, Wang X, et al. Egg cell-specific promoter-controlled CRISPR/Cas9 efficiently generates homozygous mutants for multiple target genes in Arabidopsis in a single generation. Genome Biol. 2015;16:144. doi:10.1186/s13059-015-0715-0.

41. Jia H, Wang N. Targeted genome editing of sweet orange using Cas9/ sgRNA. PLoS One. 2014;9:e93806. doi:10.1371/journal.pone.0093806.

42. Upadhyay SK, Kumar J, Alok A, Tuli R. RNA-guided genome editing for target gene mutations in wheat. G3 (Bethesda). 2013;3:2233-8. doi:10.1534/ g3.113.008847.

43. Xie K, Yang Y. RNA-guided genome editing in plants using a CRISPR-Cas system. Mol Plant. 2013;6:1975-83. doi:10.1093/mp/sst119.

44. Jacobs TB, LaFayette PR, Schmitz RJ, Parrott WA. Targeted genome modifications in soybean with CRISPR/Cas9. BMC Biotechnol. 2015;15:16 doi:10.1186/s12896-015-0131-2.

45. Ran FA, Hsu PD, Lin C, Gootenberg JS, Konermann S, Trevino AE, et al. Double nicking by RNA-guided CRISPR Cas9 for enhanced genome editing specificity. Cell. 2013;154:1380-9. doi:10.1016/j.cell.2013.08.021. 
46. Schiml S, Fauser F, Puchta H. The CRISPR/Cas system can be used as nuclease for in planta gene targeting and as paired nickases for directed mutagenesis in Arabidopsis resulting in heritable progeny. Plant J. 2014;80:1139-50. doi:10.1111/tpj.12704.

47. Puchta H, Fauser F. Gene targeting in plants: 25 years later. Int J Dev Biol. 2013;57:629-37. doi:10.1387/ijdb.130194hp.

48. Fauser F, Roth N, Pacher M, Ilg G, Sánchez-Fernández R, Biesgen C, et al. In planta gene targeting. Proc Natl Acad Sci USA. 2012;109:7535-40. doi:10.1073/pnas.1202191109.

49. Baltes NJ, Gil-Humanes J, CermakT, Atkins PA, Voytas DF. DNA replicons for plant genome engineering. Plant Cell. 2014;26:151-63. doi:10.1105/ tpc.113.119792.

50. ČermákT, Baltes NJ, Čegan R, Zhang Y, Voytas DF. High-frequency, precise modification of the tomato genome. Genome Biol. 2015;16:232. doi:10.1186/s13059-015-0796-9.

51. Ma X, Zhang Q, Zhu Q, Liu W, Chen Y, Qiu R, et al. A Robust CRISPR/ Cas9 system for convenient, high-efficiency multiplex genome editing in monocot and dicot plants. Mol Plant. 2015;8:1274-84. doi:10.1016/j. molp.2015.04.007.

52. Xie K, Minkenberg B, Yang Y. Boosting CRISPR/Cas9 multiplex editing capability with the endogenous tRNA-processing system. Proc Natl Acad Sci USA. 2015:112:3570-5. doi:10.1073/pnas.1420294112.

53. Ali Z, Abul-Faraj A, Li L, Ghosh N, Piatek M, Mahjoub A, et al. Efficient virusmediated genome editing in plants using the CRISPR/Cas9 system. Mol Plant. 2015. doi:10.1016/j.molp.2015.02.011.

54. Qi LS, Larson MH, Gilbert LA, Doudna JA, Weissman JS, Arkin AP, et al. Repurposing CRISPR as an RNA-guided platform for sequence-specific control of gene expression. Cell. 2013;152:1173-83. doi:10.1016/j. cell.2013.02.022.
55. Gilbert LA, Larson MH, Morsut L, Liu Z, Brar GA, Torres SE, et al. CRISPRmediated modular RNA-guided regulation of transcription in eukaryotes. Cell. 2013;154:442-51. doi:10.1016/j.cell.2013.06.044.

56. Piatek A, Ali Z, Baazim H, Li L, Abulfaraj A, Al-Shareef S, et al. RNA-guided transcriptional regulation in planta via synthetic dCas9-based transcription factors. Plant Biotechnol J. 2015;13:578-89. doi:10.1111/pbi.12284.

57. Tiwari SB, Belachew A, Ma SF, Young M, Ade J, Shen Y, et al. The EDLL motif: a potent plant transcriptional activation domain from AP2/ERF transcription factors. Plant J. 2012;70:855-65. doi:10.1111/j.1365-313X.2012.04935.x.

58. Morbitzer R, Römer P, Boch J, Lahaye T. Regulation of selected genome loci using de novo-engineered transcription activator-like effector (TALE)type transcription factors. Proc Natl Acad Sci USA. 2010;107:21617-22. doi:10.1073/pnas.1013133107.

59. Hiratsu K, Matsui K, Koyama T, Ohme-Takagi M. Dominant repression of target genes by chimeric repressors that include the EAR motif, a repression domain, in Arabidopsis. Plant J. 2003;34:733-9.

60. Gao Y, Zhang Y, Zhang D, Dai X, Estelle M, Zhao Y. Auxin binding protein $1(\mathrm{ABP} 1)$ is not required for either auxin signaling or Arabidopsis development. Proc Natl Acad Sci USA. 2015;112:2275-80. doi:10.1073/ pnas. 1500365112

61. Woo JW, Kim J, Kwon SI, Corvalán C, Cho SW, Kim H, et al. DNA-free genome editing in plants with preassembled CRISPR-Cas9 ribonucleoproteins. Nat Biotechnol. 2015. doi:10.1038/nbt.3389.

\section{Submit your next manuscript to BioMed Central and we will help you at every step:}

- We accept pre-submission inquiries

- Our selector tool helps you to find the most relevant journal

- We provide round the clock customer support

- Convenient online submission

- Thorough peer review

- Inclusion in PubMed and all major indexing services

- Maximum visibility for your research

Submit your manuscript at www.biomedcentral.com/submit

() Biomed Central 